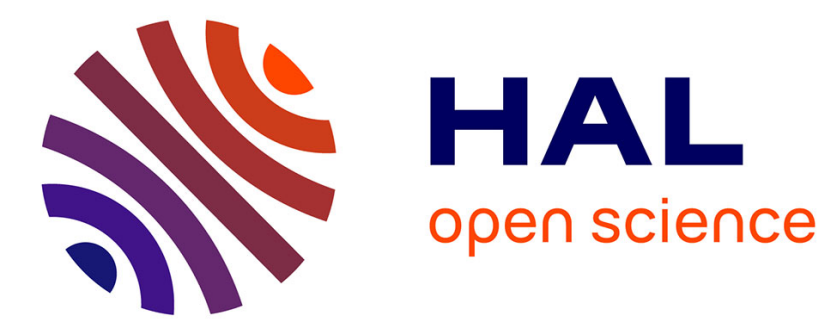

\title{
Gestion forestière et diversité biologique: un grand pas en avant (Présentation d'ouvrages).
}

Jean Pardé

\section{To cite this version:}

Jean Pardé. Gestion forestière et diversité biologique: un grand pas en avant (Présentation d'ouvrages).. 2000, pp.548-550. 10.4267/2042/5387 . hal-03443404

\author{
HAL Id: hal-03443404 \\ https://hal.science/hal-03443404
}

Submitted on 23 Nov 2021

HAL is a multi-disciplinary open access archive for the deposit and dissemination of scientific research documents, whether they are published or not. The documents may come from teaching and research institutions in France or abroad, or from public or private research centers.
L'archive ouverte pluridisciplinaire HAL, est destinée au dépôt et à la diffusion de documents scientifiques de niveau recherche, publiés ou non, émanant des établissements d'enseignement et de recherche français ou étrangers, des laboratoires publics ou privés. 


\section{GESTION FORESTIÈRE ET DIVERSITÉ BIOLOGIQUE : UN GRAND PAS EN AVANT}

\section{J. PARDÉ}

Protection des forêts en Europe, gestion forestière durable, prise en compte de la diversité biologique dans cette gestion, etc. À ces problèmes, les temps récents apportèrent une première et discrète ébauche de solution à la conférence européenne de Strasbourg, consacrée à la protection des forêts (1990).

Vinrent ensuite, plus vigoureusement, la conférence des Nations-Unies sur l'Environnement et le Développement (Rio de Janeiro, 1992), puis la conférence d'Helsinki sur le concept de gestion durable (1993, et son suivi en 1994).

On prendra bon contact avec ces événements internationaux majeurs dans les récentes parutions de la Revue forestière française (par exemple, Barthod et Touzet, 1994 ; numéro spécial "La gestion durable des forêts tempérées", 1996) et avec leurs premiers développements, souvent hésitants, sous l'impulsion des deux "directives" de la Communauté européenne, la directive Oiseaux (1979) et la directive Habitats, faune, flore (1992), par deux articles de J.-C. Rameau (1997, 1999).

Après les principes suivent maintenant leurs applications techniques et pratiques sur le terrain, bien conseillées et ordonnées par un double ouvrage $\left(^{*}\right)$, conçu et réalisé conjointement par l'Office national des Forêts, l'École nationale du Génie rural, des Eaux et des Forêts et l'Institut pour le Développement forestier (J.-C. Rameau, C. Gauberville, N. Drapier, juillet 2000).

Cette œuvre essentielle mérite maintenant présentation très positive.

$$
* *
$$

Quelques définitions d'abord.

Le but de J.-C. Rameau, C. Gauberville et N. Drapier est donc de traduire en actions de gestion les recommandations des directives Habitats et Conservation des oiseaux sauvages.

La directive de base est Habitats, signée en 1992 par l'ensemble des ministres de l'Environnement de l'Union européenne. Elle a pour objectif de favoriser la biodiversité par la conservation (voire la restauration) des habitats naturels, ainsi que de la faune et la flore sauvage qui les habitent.

Un habitat, au sens de la directive, est tout d'abord un écosystème en tant qu'ensemble indissociable comprenant un compartiment stationnel, une flore et une faune associées. Mais le terme habitat est aussi utilisé pour les espèces, et désigne alors les conditions physiques et biotiques dans lesquelles se maintient une espèce à l'état spontané. 


\section{Biologie et forêt}

La directive Habitats se traduit d'abord, sur le terrain, par la création de tout un réseau européen d'espaces où une gestion adaptée sera conduite : le réseau Natura 2000 (Rameau, 1997). L'indicateur le plus simple, et le plus sûr, d'un type d'habitat est un groupement végétal ; les groupements caractérisants retenus ont du reste fait l'objet, au plan européen, d'un recensement ordonné suivant deux codes : le code "Natura 2000", et le code "Corine biotopes", constituant une typologie européenne de référence pour les types d'habitats.

L'objectif est maintenant de publier des guides pratiques d'identification, de reconnaissance, et de gestion durable des espèces et des habitats forestiers, à intérêt communautaire.

$$
* *
$$

Viennent ainsi d'être élaborés et publiés pour la France (mais la Wallonie et le Luxembourg se sont associés à ce travail) deux "guides de gestion et diversité biologique" : l'un concerne le domaine biogéographique atlantique (sensu lato), l'autre le domaine continental (1).

Chacun de ces deux guides comprend tout d'abord un "livret général" séparé, 110-120 pages au format $16 \times 24 \mathrm{~cm}$, expliquant ce qu'est la biodiversité, quel est son intérêt, comment la prendre en compte par la notion d'habitat.

L'articulation en est la suivante :

- Chapitre 1 : Biodiversité et gestion forestière (rédigé par Ch. Barthod, 14 pages) ;

- Chapitre 2 : Directive Habitats et directive Oiseaux ;

- Chapitre 3 : Prise en compte de la diversité biologique dans la gestion ;

- Chapitre 4 : Le guide des habitats et des espèces ;

- Glossaire ;

- Bibliographie, classée par thèmes (20 pages).

Le guide proprement dit se présente sous forte couverture et grand format. II est composé d'une suite de fiches, solides et mobiles. Le plus souvent, à une fiche recto verso est consacré un habitat, décrit avec précision et clarté aux plans écologique, biologique et dynamique, sans oublier les rubriques "potentialités" et "itinéraires sylvicoles", ni même, le cas échéant, les plantations résineuses.

Les habitats forestiers sont regroupés par "grands types", dont voici un exemple :

À un grand type, "Hêtraies", est consacrée une fiche initiale, qui en donne les caractères généraux. Viennent ensuite des fiches précisant les structures principales des différents "sous-types", tel celui dénommé : "hêtraies sèches". Suivent enfin les habitats élémentaires et, par exemple, les "hêtraieschênaies calcicoles sèches du centre-est du Bassin parisien".

Aux habitats exclusivement forestiers succèdent, moins détaillés (on ne descend pas jusqu'aux habitats élémentaires), les habitats simplement associés : telles les "formations à Genévrier commun" ou les "complexes de tourbières".

Aux fiches "habitats forestiers, ou associés" succèdent les fiches "espèces", une fiche recto verso par espèce, animale ou végétale, d'intérêt communautaire, et dont la conservation nécessite la désignation de zones spéciales de conservation : des mammifères (le castor, par exemple) aux oiseaux (la chouette de Tegmalm, par exemple), aux amphibiens, jusqu'aux espèces végétales, tel

(1) Restent deux domaines à traiter de la même façon : le domaine biogéographique "hautes montagnes" (qui couvre les Alpes "centrales" et les Pyrénées "centrales") et le domaine méditerranéen. 


\section{J. PARDÉ}

le sabot de Vénus : toutes espèces - et elles seules, certaines étant même prioritaires - dont il convient de protéger spécifiquement l'habitat.

$$
\text { *** }
$$

La création de ces guides a rassemblé beaucoup de compétences diverses et de travail en commun.

Leur application devrait maintenant permettre d'assurer au mieux une gestion multifonctionnelle, réunissant :

- la fonction de production de la forêt,

- sa fonction de protection (des sols, des ressources en eau),

- sa fonction patrimoniale,

- et sa fonction sociale.

J. PARDÉ

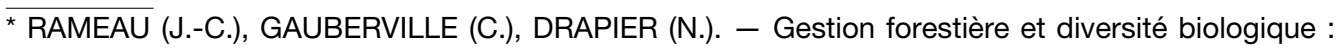
identification et gestion intégrée des habitats et espèces d'intérêt communautaire comportant :

- un livret général sur la biodiversité, 119 pages, format 15,5 x $24 \mathrm{~cm}$,

- un premier "guide" sous forme de classeur composé de 140 fiches "habitat" + 49 fiches "espèce" mobiles, format $32,5 \times 24 \mathrm{~cm}$, plusieurs centaines d'illustrations en couleurs et schémas concernant le domaine atlantique français,

- un deuxième "guide" semblable, de 113 fiches "habitat" et 47 fiches "espèce", concernant le domaine continental français.

Chaque ensemble "livret général + classeur" est vendu au prix de $480 \mathrm{~F}$ (plus frais d'envoi $35 \mathrm{~F}$ pour un ou deux exemplaires ; $50 \mathrm{~F}$ au-delà) par IDF Diffusion - 23, avenue Bosquet - F-75007 PARIS. Tél. 01.40.62.22.81. Télécopie : 01.45.55.98.54. Mél. paris@association-idf.com

\section{BIBLIOGRAPHIE}

BARTHOD (Ch.), TOUZET (G.). - De Strasbourg à Helsinki, les deux premières conférences ministérielles pour la protection des forêts en Europe. - Revue forestière française, vol. XLVI, n 4, 1994, pp. 319-334.

RAMEAU (J.-C.). - La Directive Habitats : analyse d'un échec, réflexions pour l'avenir. - Revue forestière française, $n^{\circ} 5,1997$, pp. 399-416.

RAMEAU (J.-C.). - Aménagement forestier, importance de l'écologie, prise en compte de la biodiversité. Revue forestière française, vol. LI, n spécial “L'Aménagement forestier, hier, aujourd'hui, demain”, 1999, pp. 87-101. 